\title{
RESPIRATORY DISTRESS AND PROPHYLAXIS WITH BETAMETHASONE IN LATE PRETERM BABIES: A RETROSPECTIVE STUDY
}

\author{
D. Ferrari, C. Poggiani \\ NICU, Istituti Ospitalieri, Cremona, Italy
}

Background and aims: The occurrence of respiratory distress (RDS) in infants born at $340 / 7$ through $366 / 7$ weeks' gestation or "late-preterm" (LP) is understudied. This study aimed to examine the incidence of RDS in late preterm infants admitted to a NICU and additionally to analyse the effect of maternal prophylaxis with betamethasone on RDS.

Methods: A retrospective cohort of neonates born at 34 0/7 through 36 6/7 weeks' gestational age and admitted at birth to a NICU of a tertiary hospital between January 2006 and December 2010. RDS diagnosis was recorded and classified as mild-moderate and severe. Clinical variables and length of stay (LOS) were collected. The association between prophylaxis with betamethasone before 34 weeks gestational age (GA) and RDS was tested in univariate analysis.

Results: A total of 322 newborn LP were included, with a mean GA of $35.2 \pm 0.6$ weeks and a mean birth weight of $1.989 \pm 87 \mathrm{~g}$. The mean LOS in the NICU was $10 \pm 3 \mathrm{~g}$. The incidence of RDS was $30 \% ; 10 \mathrm{LP}$ (11.1\%) had severe RDS with mechanical ventilation, while 17 LP (18.6\%) had mild-moderate RDS requiring continuous positive air pressure. In the group not treated with betamethasone the incidence of RDS $(23 \%)$ was significantly higher than in the group treated with betamethasone $(6.2 \%)(p<0.029)$.

Conclusions: RDS is frequent in LP. Maternal prophylaxis with betamethasone is associated with lower incidence of RDS. Studies are warranted to determine the beneficial effect of bethametasone prophylaxis on RDS in this population. 